This document was prepared in conjunction with work accomplished under Contract No. DE-AC09-96SR18500 with the U. S. Department of Energy.

\title{
DISCLAIMER
}

This report was prepared as an account of work sponsored by an agency of the United States Government. Neither the United States Government nor any agency thereof, nor any of their employees, nor any of their contractors, subcontractors or their employees, makes any warranty, express or implied, or assumes any legal liability or responsibility for the accuracy, completeness, or any third party's use or the results of such use of any information, apparatus, product, or process disclosed, or represents that its use would not infringe privately owned rights. Reference herein to any specific commercial product, process, or service by trade name, trademark, manufacturer, or otherwise, does not necessarily constitute or imply its endorsement, recommendation, or favoring by the United States Government or any agency thereof or its contractors or subcontractors. The views and opinions of authors expressed herein do not necessarily state or reflect those of the United States Government or any agency thereof. 
Keywords:

Tank Farm

Characterization

Saltstone WAC

Retention Time: Permanent

\section{Characterization of Tank 23H Supernate Per Saltstone Waste Acceptance Criteria Analysis Requirements-2005}

Author: L. N. Oji and M. S. Blume

Publication date: May 26, 2005

Westinghouse Savannah River Company Savannah River Site Aiken, SC 29808

Prepared for the U. S. Department of Energy Under Contract Number DE-AC09-96SR18500

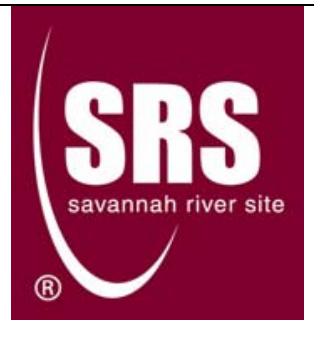


WSRC-TR-2005-00192, Rev. 1

June 1, 2005

REVIEWS AND APPROVALS

AUTHOR(S)

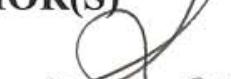

asthenes

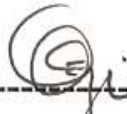

$6 / 1 / 05$

L. N. Oji, Waste Processing Technology

Date
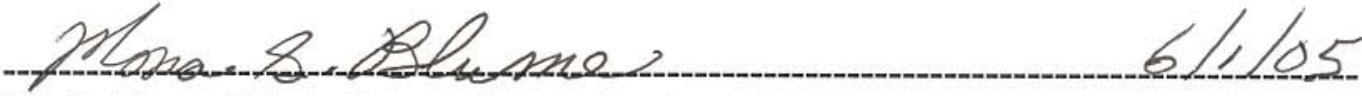

M. S. Blume, Waste Processing Technology

Date

Design Check per Manual E7, Procedure 2.60

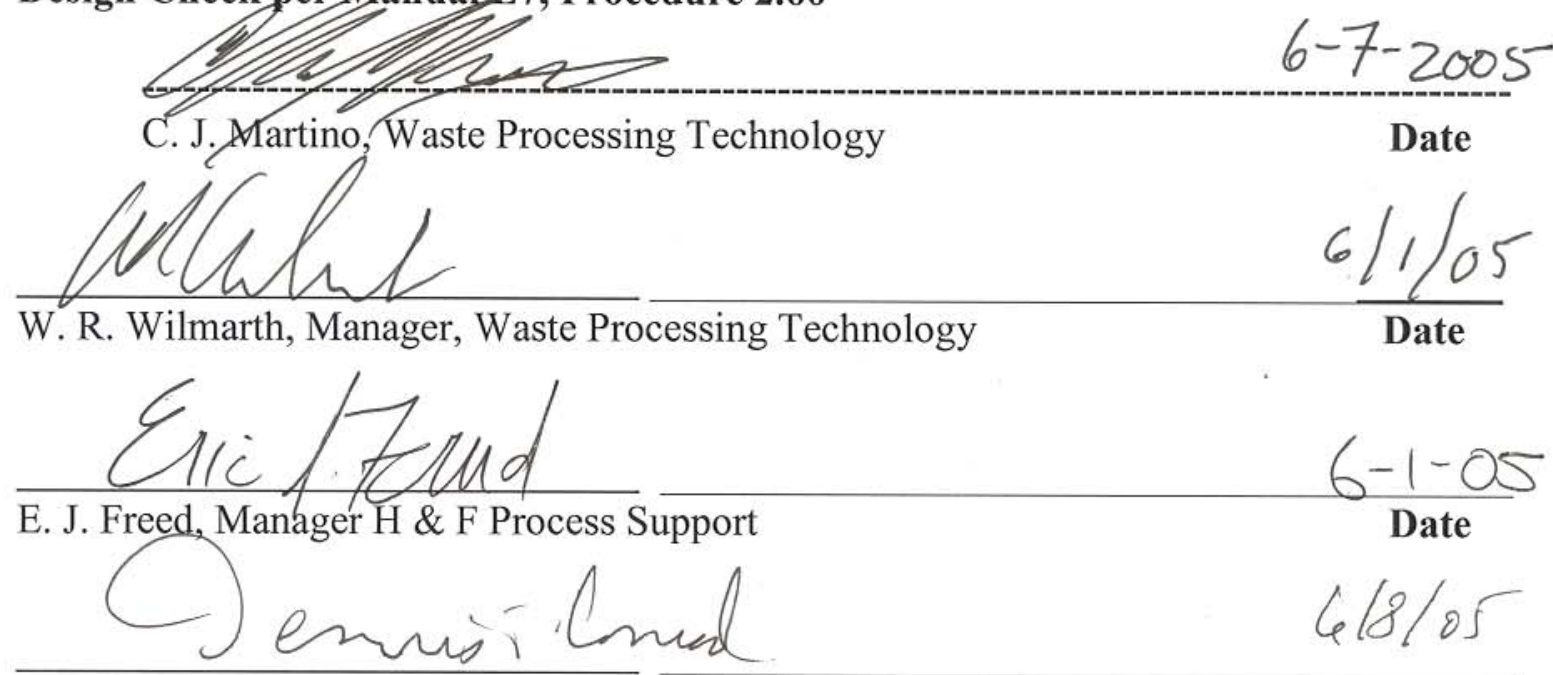

D. T. Conrad, Manager, Salt Engineering

Date

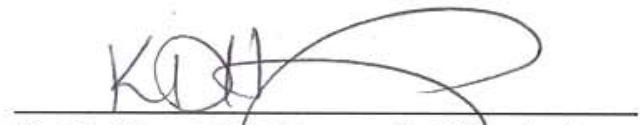

K. D. Harp, Manager, Salt Projects

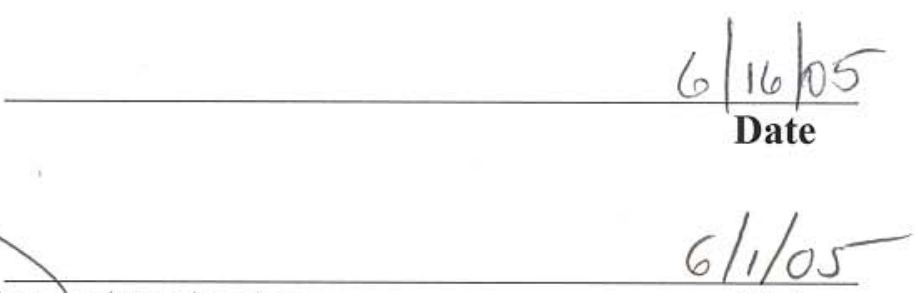

P. J. Hill, Manager, Planning Integration and Technology

Date

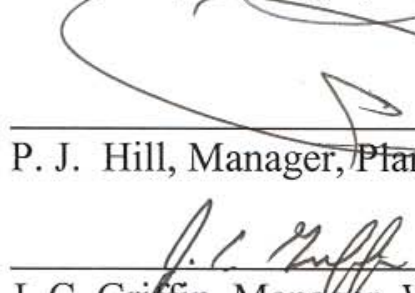

J. C. Griffin, Manager, Waste Processing Technology

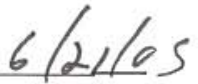


WSRC-TR-2005-00192, Rev. 1

May 5, 2005

\section{SUMMARY}

Page 3 of 11

Variable depth Tank 23H samples (22-inch sample [HTF-014] and 185-inch sample [HTF-013]) were pulled from Tank $23 \mathrm{H}$ in February, 2005 for characterization. The characterization of the Tank $23 \mathrm{H}$ low activity waste is part of the overall liquid waste processing activities. This characterization examined the species identified in the Saltstone Waste Acceptance Criteria (WAC) for the transfer of waste into the Salt-Feed Tank (SFT). The samples were delivered to the Savannah River National Laboratory (SRNL) and analyzed.

Apart from radium-226 with an average measured detection limit of $<2.64 \mathrm{E}+03 \mathrm{pCi} / \mathrm{mL}$, which is about the same order of magnitude as the WAC limit $(<8.73 \mathrm{E}+03 \mathrm{pCi} / \mathrm{mL})$, none of the species analyzed was found to approach the limits provided in the Saltstone WAC. The concentration of most of the species analyzed for the Tank $23 \mathrm{H}$ samples were 2-5 orders of magnitude lower than the WAC limits. The achievable detection limits for a number of the analytes were several orders of magnitude lower than the WAC limits, but one or two orders of magnitude higher than the requested detection limits. Analytes which fell into this category included plutonium-241, europium-154/155, antimony-125, tin-126, ruthenium/rhodium-106, selenium-79, nickel-59/63, ammonium ion, copper, total nickel, manganese and total organic carbon.

\section{INTRODUCTION}

The Saltstone Production Facility (SPF) located in Z Area is permitted as a wastewater treatment plant per the South Carolina Pollution Control Act, Title 48, Chapter 1. The aqueous waste is received and treated in the SPF to produce saltstone grout that is then transferred to the Saltstone Disposal Facility (SDF) for final disposal. As presently permitted by the South Carolina Department of Health and Environmental Control (SCDHEC), all transfers of aqueous waste to the SPF shall come through the jacketed pipeline that connects the Tank $50 \mathrm{H}$ with the Salt Feed Tank (SFT) in Z Area. The Acceptance Criteria for Aqueous Waste Sent to the Z-area Saltstone Production Facility ${ }^{1}$ describes analytical checks required prior to transferring aqueous waste from Tank $50 \mathrm{H}$ to the Saltstone Production Facility. Aqueous waste meeting the WAC limits can be safely transferred, stored and treated in the SPF for subsequent disposal as saltstone in the SDF.

Closure Business Unit (CBU) personnel requested analyses on a sample of Tank $23 \mathrm{H}$ waste which was pulled on February 15, 2005. The characterization of the Tank $23 \mathrm{H}$ low activity waste is part of the overall liquid waste processing activities aimed at the removal and aggregation of the Tank $49 \mathrm{H}$ salt solution in Tank $50 \mathrm{H}$ and final disposal through the Saltstone Facility of approximately 1,255,000 gallons of low activity waste currently stored in the tank. Thus, before sending low activity Tank $23 \mathrm{H}$ material to Tank $50 \mathrm{H}$ for

\footnotetext{
1 "Acceptance Criteria for Aqueous Waste Sent to the Z Area Saltstone Production Facility (U)," X-SD-Z-00001, Rev. 2, September7,2004
} 
WSRC-TR-2005-00192, Rev. 1

May 5, 2005

Page 4 of 11

aggregation purposes, specific measured values for the WAC constituents are required to determined a Tank $23 \mathrm{H}$ baseline composition in the aggregation blending evaluation.

The two types of Tank $23 \mathrm{H}$ variable depth supernate samples sent to SRNL were contained in either stainless steel dip bottles or liter-size poly-bottles shipped in paint cans. This report describes the work performed to analyze the WAC constituents in the sample pulled from Tank 23H per Technical Task Request number SP-TTR-2004-00025, Rev.1.

\section{EXPERIMENTAL}

Of the six stainless steel (SS) bottles containing Tank $23 \mathrm{H}$ variable depth samples supplied for organic characterization, three (all identified as HTF-014) contained samples from the 22-inch level of the Tank $23 \mathrm{H}$ and the remainder from the 185 -inch level of the tank (all identified as HTF-013). Another set of Tank 23H samples were also provided in poly-bottles. These poly-bottles samples contained supernate liquid of approximately 400 $\mathrm{mL}$ and were also from the two variable depth samples as described above (HTF-014 and HTF-013). One of the poly-bottle samples, the 22-inch sample (HTF-014), contained some visible solids. See Figure 1 inserts A-C. In consultation with the customer, the solids were filtered off using a 0.45 micron nylon filter prior to compositing of the contents of the poly-bottles. The Tank $23 \mathrm{H}$ solids recovered after filtration were less than $1 \%$ by volume of the total sample and were not included in the analysis.

Equal volumes of the SS samples from the 22-inch sample (HTF-014) and 185-inch sample (HTF-013) were composited for organic characterization and the remainder combined with the composited samples from the poly-bottles. (While the use of stainless steel bottles does not fit the EPA protocol for handling samples that potentially contain VOCs, these are used instead of glass vials because of safety considerations with radioactive samples.) This brought the total volume of the Tank $23 \mathrm{H}$ composite sample supplied for analysis to approximately 1.8 Liters. The composited sample was divided and submitted to the SRNL Analytical Development Section (ADS) for the required analyses. These samples were analyzed for the analytes specified in Attachments 8.1, 8.2 and 8.3 of the Acceptance Criteria for Aqueous Waste Sent to the Z-area Saltstone Production Facility (WAC) (X-SD-Z-00001, Rev. 2). These analytes are given in Tables 1 and 2 along with the requested detection limit targets and WAC acceptance limits. While target minimum detection limit for each analyte were identified (See target detection limits in Tables -2), ADS personnel were requested to tailor their procedures to attempt meeting the requested limits. All results reported are from the duplicate composite sample (samples 1 and 2).

A number of different analytical methods were used by ADS to determine the concentrations of various species in the samples. Ion chromatography (IC) is used to measure a number of anion species $\left(\mathrm{NO}_{3}^{-}, \mathrm{NO}_{2}^{-}, \mathrm{SO}_{4}{ }^{--}, \mathrm{F}^{-}, \mathrm{Cl}^{-}, \mathrm{C}_{2} \mathrm{O}_{4}{ }^{--}\right.$and $\left.\mathrm{PO}_{4}{ }^{--}\right)$as well as the cation $\mathrm{NH}_{4}{ }^{+}$. Carbonate $\left(\mathrm{CO}_{3}{ }^{-}\right)$and free hydroxide were determined by a wet chemistry titration method. Inductively coupled plasma-emission spectrometry (ICP-ES) is used to determine most of the transition metals ( $\mathrm{Ag}, \mathrm{B}, \mathrm{Ba}, \mathrm{Be}, \mathrm{Ca}, \mathrm{Cd} . \mathrm{Cr}, \mathrm{Cu}, \mathrm{Mg}$, 
$\mathrm{Mn}, \mathrm{Na}, \mathrm{Ni}, \mathrm{Pb}, \mathrm{Sb}, \mathrm{Si}$, and $\mathrm{Zn}$ and others). Atomic Absorption (AA) is used to determine As, Se and K. Acid digestion followed by cold vapor AA (CVAA) technique is used to measure the $\mathrm{Hg}$ concentration. Both volatile and semivolatile organic species are determined using gas chromatography coupled with mass spectrometry (GC/MS). Ethylenediamine tetra-acetic acid (EDTA) was determined using ion pair chromatography (IPC). Gas chromatography combined with mass spectrometry (GC/MS) was used to check for most volatile (benzene, butanol and isopropanol) and semi-volatile organic compounds (tributyl phosphate and phenol). There is currently no method available for radioactive samples for methanol analysis. ADS used high pressure liquid chromatography (HPLC) to analyze for sodium tetraphenylborate. Total organic carbon (TOC) was determined using a total organic carbon analyzer. Total suspended solids were measured gravimetrically. A $\mathrm{pH}$ meter was used to measure sample $\mathrm{pH}$. Inductively coupled plasma-mass spectrometry (ICP-MS) is used to characterize for ${ }^{237} \mathrm{~Np},{ }^{99} \mathrm{Tc}$, the uranium isotopes and fission products.

Radionuclide determinations were also made using a number of different methods. Gamma spectrometry was used where possible to determine many radionuclides $\left({ }^{137} \mathrm{Cs}\right.$, ${ }^{60} \mathrm{Co},{ }^{94} \mathrm{Nb},{ }^{106} \mathrm{Ru} / \mathrm{Rh},{ }^{126} \mathrm{Sn},{ }^{125} \mathrm{Sb},{ }^{134} \mathrm{Cs},{ }^{154} \mathrm{Eu},{ }^{155} \mathrm{Eu},{ }^{144} \mathrm{Ce}$ and ${ }^{94} \mathrm{Nb}$,). Am/Cm analysis was used to analyze for ${ }^{241} \mathrm{Am},{ }^{242 \mathrm{~m}} \mathrm{Am}$ and ${ }^{243} \mathrm{Cm}$. Gross alpha and non-volatile beta determinations were made by first removing the tritium and then using liquid scintillation counting. ${ }^{59} \mathrm{Ni},{ }^{63} \mathrm{Ni},{ }^{90} \mathrm{Sr},{ }^{79} \mathrm{Se},{ }^{14} \mathrm{C},{ }^{129} \mathrm{I}$, and ${ }^{241} \mathrm{Pu}$ were determined by chemical separations followed by beta counting. Tritium was determined by beta counting after separating the tritium by distillation.

It should be noted that the SRNL is not an EPA-approved analytical laboratory for regulatory samples.

\section{Results of Analyses of Tank 23H WAC Samples}

The results of the analyses provided in the tables below for the Tank $23 \mathrm{H}$ WAC characterizations are for duplicate analytical determinations by SRNL Analytical Development Section (ADS). For many species the concentration fell below the lower limit of detection. In these cases, ADS reported the lower limit of detection preceded by " $<$ ". Tables 1 and 2 provide, respectively, the measured values for chemical contaminants and radionuclides for the Tank $23 \mathrm{H}$ samples from the composited variable depth samples (22-inch sample [HTF-014] and 185-inch sample [HTF-013]) pulled in February of 2005, along with the given WAC limits and the plant requested detection limits.

Apart from radium-226 with an average measured concentration of $<2.64 \mathrm{E}+03 \mathrm{pCi} / \mathrm{mL}$, which is about the same order of magnitude as the WAC limit $(<8.73 \mathrm{E}+03 \mathrm{pCi} / \mathrm{mL})$, none of the species analyzed was found to exceed the limits provided in the Saltstone WAC. The concentration of most of the species analyzed for the Tank $23 \mathrm{H}$ samples were 2-5 orders of magnitude lower than the WAC limits.

It was difficult in some cases to meet the requested detection limits for some species because of dilution and matrix effects problems, especially in those cases where the requested detection limits were quite low and approached the theoretical instrument 
WSRC-TR-2005-00192, Rev. 1

May 5, 2005

Page 6 of 11

detection limits with dilution of the samples. This happens to be the case with a number of the analytes whose achievable detection limits were several orders of magnitude lower than the WAC limits, but one or two orders of magnitude higher than the requested limits of detection. Analytes which fell into this category included plutonium-241, europium154/155, antimony-125, tin-126, ruthenium/rhodium-106, selenium-79, nickel-59/63 ammonium ion, and total organic carbon. The results reported for plutonium-240 are based on $20 \%$ of the value obtained for plutonium-239/240 analysis. In those instances when some transition metals $(\mathrm{Cd}, \mathrm{Pb}, \mathrm{Ag}$ and $\mathrm{Mo})$, originally slated to be analyzed by ICP-ES, failed to meet the requested limit due to salt effects the concentration of these metals where obtained directly from the fission product characterization of the samples by ICP-MS. All but a few of the transition metals $(\mathrm{Mn}, \mathrm{Ni}$ and $\mathrm{Cu}$ ) met the requested detection limits. Those which did not meet these limits were within about an order of magnitude higher.

Some radionuclides originally requested for individual analysis in Tank $23 \mathrm{H}$ samples $\left({ }^{152} \mathrm{Eu},{ }^{227} \mathrm{Ac},{ }^{249} \mathrm{Bk},{ }^{229} \mathrm{Th},{ }^{231} \mathrm{~Pa},{ }^{232} \mathrm{U}\right.$-mostly minor beta/gamma and alpha emitters) were only bounded by the total alpha and beta/gamma values. In a verbal request, during the Tank $23 \mathrm{H}$ analytical characterization process, the plant customer identified these bounding values as sufficient and requested termination of the above radionuclides from further analysis.

The requested limits (last column of Tables 1 and 2) are updated values of the original requested limits as found in the technical task plan (TTP) for this Tank 23H characterizations (WSRC-RP-2005-01299, Rev. 1). Some of the new updated request limits are several orders of magnitude lower than the original requested limits for most of the species analyzed. 
WSRC-TR-2005-00192, Rev. 1

May 5, 2005

Page 7 of 11

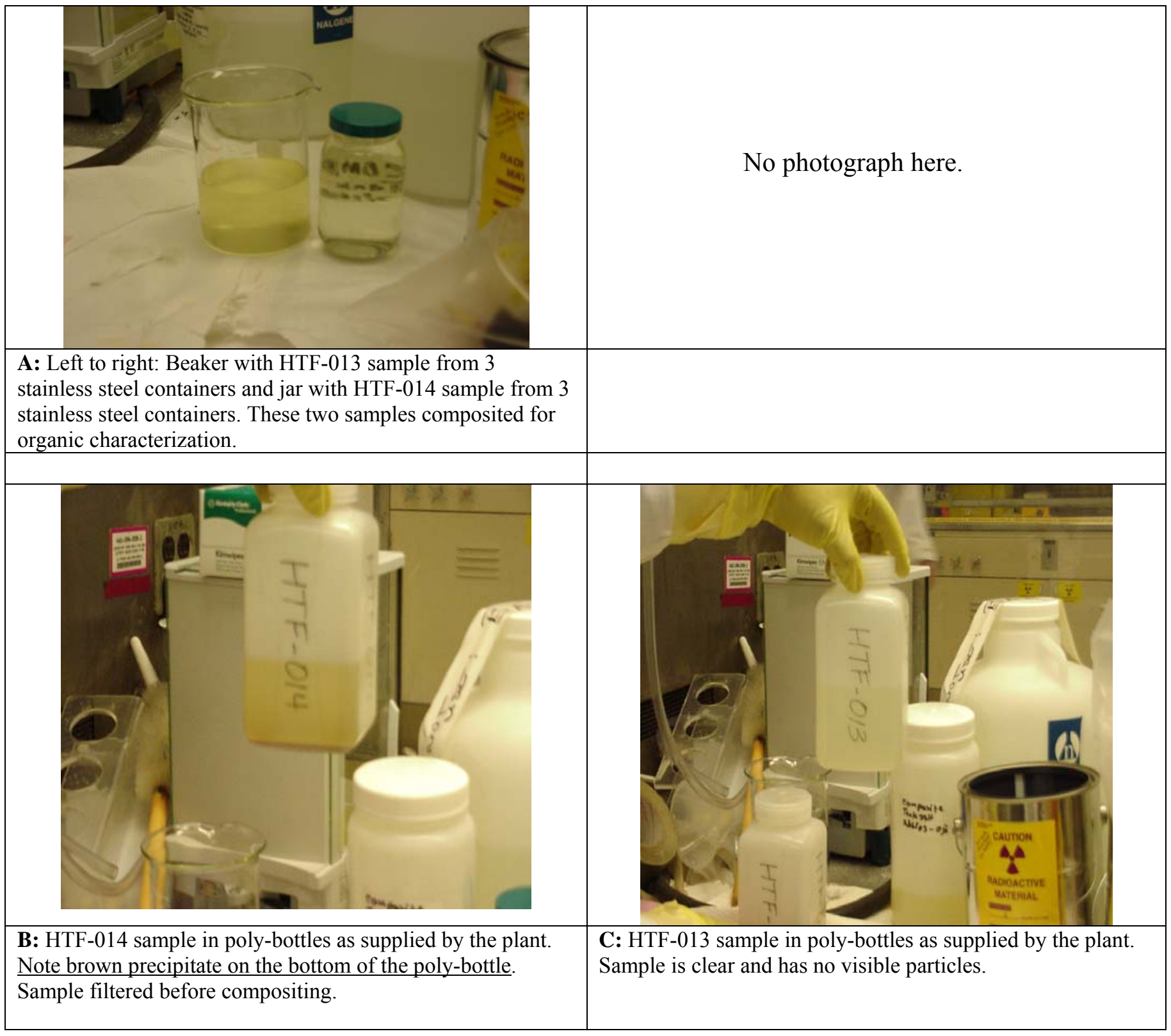

Figure 1. Photographs of Tank $23 \mathrm{H}$ variable depth samples from the 22 (HTF-014) and 185-inch (HTF-013) levels of the tank. 
WSRC-TR-2005-00192, Rev. 1

May 5, 2005

Page 8 of 11

Table 1. Tank 23H WAC Sample Results for Chemical Contaminants (Units of mg/L except where noted).

\begin{tabular}{|c|c|c|c|c|c|c|}
\hline Chemical Species & Sample 1 & Sample 2 & Average & $\begin{array}{l}\text { Stand } \\
\text { deviation }\end{array}$ & WAC Limit & $\begin{array}{l}\text { Requested } \\
\text { Detection Limit }\end{array}$ \\
\hline \multicolumn{7}{|l|}{ Solvated Ions } \\
\hline Ammonium $\left(\mathrm{NH}_{4}^{+}\right)$ & $<1.0 \mathrm{E}+01$ & $<1.0 \mathrm{E}+01$ & $<1.0 \mathrm{E}+01$ & - & $7.13 \mathrm{E}+03$ & $7.13 \mathrm{E}+00$ \\
\hline Carbonate $\left(\mathrm{CO}_{3}^{-2}\right)$ & $2.72 \mathrm{E}+03$ & $2.69 \mathrm{E}+03$ & $2.71 \mathrm{E}+03$ & $1.70 \mathrm{E}+01$ & $1.45 \mathrm{E}+05$ & $1.45 \mathrm{E}+02$ \\
\hline Chloride $\left(\mathrm{Cl}^{-}\right)$ & $4.00 \mathrm{E}+00$ & $4.00 \mathrm{E}+00$ & $4.00 \mathrm{E}+00$ & $0.00 \mathrm{E}+00$ & $9.68 \mathrm{E}+03$ & $9.68 \mathrm{E}+00$ \\
\hline Fluoride $\left(\mathrm{F}^{-}\right)$ & $2.00 \mathrm{E}+00$ & $2.00 \mathrm{E}+00$ & $2.00 \mathrm{E}+00$ & $0.00 \mathrm{E}+00$ & $4.94 \mathrm{E}+03$ & $4.94 \mathrm{E}+00$ \\
\hline Hydroxide $\left(\mathrm{OH}^{-}\right)$ & $3.32 \mathrm{E}+03$ & $2.94 \mathrm{E}+03$ & $3.13 \mathrm{E}+03$ & $2.64 \mathrm{E}+02$ & $1.91 \mathrm{E}+05$ & $1.91 \mathrm{E}+03$ \\
\hline Nitrate $\left(\mathrm{NO}_{3}{ }^{-}\right)$ & $2.44 \mathrm{E}+03$ & $2.44 \mathrm{E}+03$ & $2.44 \mathrm{E}+03$ & $0.00 \mathrm{E}+00$ & $5.29 \mathrm{E}+05$ & $5.29 \mathrm{E}+02$ \\
\hline Nitrite $\left(\mathrm{NO}_{2}^{-}\right)$ & $9.10 \mathrm{E}+03$ & $8.92 \mathrm{E}+03$ & $9.01 \mathrm{E}+03$ & $1.27 \mathrm{E}+02$ & $2.59 \mathrm{E}+05$ & $2.59 \mathrm{E}+02$ \\
\hline Oxalate $\left(\mathrm{C}_{2} \mathrm{O}_{4}^{-2}\right)$ & $1.00 \mathrm{E}+01$ & $1.10 \mathrm{E}+01$ & $1.05 \mathrm{E}+01$ & $7.07 \mathrm{E}-01$ & $3.30 \mathrm{E}+04$ & $3.30 \mathrm{E}+01$ \\
\hline Phosphate $\left(\mathrm{PO}_{4}^{-3}\right)$ & $<1.00 \mathrm{E}+01$ & $<1.00 \mathrm{E}+01$ & $<1.00 \mathrm{E}+01$ & - & $3.56 \mathrm{E}+04$ & $3.56 \mathrm{E}+01$ \\
\hline Sulfate $\left(\mathrm{SO}_{4}^{-2}\right)$ & $9.20 \mathrm{E}+01$ & $9.30 \mathrm{E}+01$ & $9.25 \mathrm{E}+01$ & 7.07E-01 & $6.89 \mathrm{E}+04$ & $6.89 \mathrm{E}+01$ \\
\hline \multicolumn{7}{|l|}{ RCRA Hazardous Metals } \\
\hline Arsenic (As) & $<2.80 \mathrm{E}-02$ & $<2.80 \mathrm{E}-02$ & $<2.80 \mathrm{E}-02$ & - & $7.50 \mathrm{E}+02$ & $1.65 \mathrm{E}-01$ \\
\hline Barium $(\mathrm{Ba})$ & 2.07E-01 & $2.47 \mathrm{E}-01$ & $2.27 \mathrm{E}-01$ & $2.83 \mathrm{E}-02$ & $7.50 \mathrm{E}+02$ & $9.00 \mathrm{E}-03$ \\
\hline Cadmium $(\mathrm{Cd})$ & $<1.64 \mathrm{E}-02$ & $<1.28 \mathrm{E}-02$ & $<1.46 \mathrm{E}-02$ & - & $3.75 \mathrm{E}+02$ & $2.50 \mathrm{E}-02$ \\
\hline Chromium (Cr) & $4.09 \mathrm{E}+00$ & $4.01 \mathrm{E}+00$ & $4.05 \mathrm{E}+00$ & $5.66 \mathrm{E}-02$ & $1.50 \mathrm{E}+03$ & $1.00 \mathrm{E}-01$ \\
\hline Lead $(\mathrm{Pb})$ & $3.01 \mathrm{E}-01$ & $2.96 \mathrm{E}-01$ & $2.98 \mathrm{E}-01$ & 3.08E-03 & $7.50 \mathrm{E}+02$ & $6.00 \mathrm{E}-01$ \\
\hline Mercury (Hg) & $1.05 \mathrm{E}+01$ & $9.85 \mathrm{E}+00$ & $1.02 \mathrm{E}+01$ & $4.60 \mathrm{E}-01$ & $3.25 \mathrm{E}+02$ & $1.00 \mathrm{E}-01$ \\
\hline Selenium $(\mathrm{Se})$ & $6.5 \mathrm{E}-02$ & $6.7 \mathrm{E}-02$ & $6.60 \mathrm{E}-02$ & $1.41 \mathrm{E}-03$ & $4.50 \mathrm{E}+02$ & $1.00 \mathrm{E}-02$ \\
\hline Silver (Ag) & $6.97 \mathrm{E}-03$ & $1.66 \mathrm{E}-03$ & $4.31 \mathrm{E}-03$ & $3.76 \mathrm{E}-03$ & $7.50 \mathrm{E}+02$ & $1.10 \mathrm{E}-02$ \\
\hline \multicolumn{7}{|l|}{ Other Metals } \\
\hline Aluminum $\left(\mathrm{Al}^{3+}\right)$ & $1.11 \mathrm{E}+01$ & $1.10 \mathrm{E}+01$ & $1.11 \mathrm{E}+01$ & 7.07E-02 & $1.41 \mathrm{E}+05$ & $1.00 \mathrm{E}+00$ \\
\hline Boron $(\mathrm{B})$ & $1.48 \mathrm{E}+00$ & $1.57 \mathrm{E}+00$ & $1.53 \mathrm{E}+00$ & $6.36 \mathrm{E}-02$ & $9.00 \mathrm{E}+02$ & $5.00 \mathrm{E}-02$ \\
\hline Calcium (Ca) & $1.48 \mathrm{E}+00$ & $2.79 \mathrm{E}+00$ & $2.14 \mathrm{E}+00$ & $9.26 \mathrm{E}-01$ & $2.76 \mathrm{E}+03$ & $1.00 \mathrm{E}-01$ \\
\hline Cobalt $(\mathrm{Co})$ & $1.87 \mathrm{E}-03$ & $1.17 \mathrm{E}-03$ & $1.52 \mathrm{E}-03$ & $4.98 \mathrm{E}-04$ & $9.00 \mathrm{E}+02$ & $9.00 \mathrm{E}-01$ \\
\hline Copper $(\mathrm{Cu})$ & $<2.00 \mathrm{E}-01$ & $<2.00 \mathrm{E}-01$ & $<2.00 \mathrm{E}-01$ & - & $9.00 \mathrm{E}+02$ & $2.00 \mathrm{E}-02$ \\
\hline Iron $(\mathrm{Fe})$ & $6.18 \mathrm{E}-01$ & $1.61 \mathrm{E}+00$ & $1.11 \mathrm{E}+00$ & $7.01 \mathrm{E}-01$ & $6.00 \mathrm{E}+03$ & $9.00 \mathrm{E}-02$ \\
\hline Lithium (Li) & $1.20 \mathrm{E}+00$ & $1.39 \mathrm{E}+00$ & $1.30 \mathrm{E}+00$ & $1.34 \mathrm{E}-01$ & $9.00 \mathrm{E}+02$ & $3.00 \mathrm{E}-02$ \\
\hline Manganese $(\mathrm{Mn})$ & $<4.00 \mathrm{E}-02$ & $<4.00 \mathrm{E}-02$ & $<4.00 \mathrm{E}-02$ & - & $9.00 \mathrm{E}+02$ & $3.00 \mathrm{E}-03$ \\
\hline Molybdenum (Mo) & $<5.84 \mathrm{E}-02$ & $<5.06 \mathrm{E}-02$ & $<5.45 \mathrm{E}-02$ & - & $9.00 \mathrm{E}+02$ & $2.00 \mathrm{E}-01$ \\
\hline Nickel (Ni) & $<1.86 \mathrm{E}+00$ & $<1.86 \mathrm{E}+00$ & $<1.86 \mathrm{E}+00$ & - & $9.00 \mathrm{E}+02$ & $3.20 \mathrm{E}-02$ \\
\hline Potassium $(\mathrm{K})$ & $2.32 \mathrm{E}+01$ & $2.60 \mathrm{E}+01$ & $2.46 \mathrm{E}+01$ & $1.98 \mathrm{E}+00$ & $3.67 \mathrm{E}+04$ & $1.00 \mathrm{E}+01$ \\
\hline Silicon $(\mathrm{Si})$ & $1.61 \mathrm{E}+02$ & $1.63 \mathrm{E}+02$ & $1.62 \mathrm{E}+02$ & $1.41 \mathrm{E}+00$ & $1.29 \mathrm{E}+04$ & $1.00 \mathrm{E}+01$ \\
\hline Sodium $(\mathrm{Na})$ & $1.22 \mathrm{E}+04$ & $1.27 \mathrm{E}+04$ & $1.25 \mathrm{E}+04$ & $3.54 \mathrm{E}+02$ & $1.61 \mathrm{E}+05$ & $1.00 \mathrm{E}+02$ \\
\hline Strontium $(\mathrm{Sr})$ & $2.69 \mathrm{E}-01$ & $6.08 \mathrm{E}-01$ & 4.39E-01 & $2.40 \mathrm{E}-01$ & $9.00 \mathrm{E}+02$ & $2.00 \mathrm{E}-02$ \\
\hline Zinc (Zn) & $2.02 \mathrm{E}+00$ & $2.10 \mathrm{E}+00$ & $2.06 \mathrm{E}+00$ & $5.66 \mathrm{E}-02$ & $9.75 \mathrm{E}+02$ & $2.00 \mathrm{E}-01$ \\
\hline Specific gravity & $1.03 \mathrm{E}+00$ & $1.05 \mathrm{E}+00$ & $1.04 \mathrm{E}+00$ & $1.41 \mathrm{E}-02$ & - & - \\
\hline $\mathrm{pH}$ (pH units) & $1.32 \mathrm{E}+01$ & $1.29 \mathrm{E}+01$ & $1.31 \mathrm{E}+01$ & $2.12 \mathrm{E}-01$ & - & - \\
\hline $\begin{array}{l}\text { Mean particle size } \\
\text { (microns) }\end{array}$ & $7.55 \mathrm{E}+01$ & $5.50 \mathrm{E}+01$ & $6.53 \mathrm{E}+01$ & $1.45 \mathrm{E}+01$ & - & - \\
\hline Suspended Solids (wt \%) & $5.00 \mathrm{E}-02$ & $<1.00 \mathrm{E}-03$ & $<2.55 \mathrm{E}-02$ & - & $\begin{array}{c}15 \mathrm{wt} \% \\
(1.88 \mathrm{E}+05 \mathrm{mg} / \mathrm{L})\end{array}$ & - \\
\hline
\end{tabular}


WSRC-TR-2005-00192, Rev. 1

May 5, 2005

Page 9 of 11

Table 1. Tank 23H WAC Sample Results for Chemical Contaminants (Units of mg/L) continued.

\begin{tabular}{|c|c|c|c|c|c|c|}
\hline Chemical Species & Sample 1 & Sample 2 & Average & $\begin{array}{l}\text { Stand } \\
\text { deviation }\end{array}$ & WAC Limit & $\begin{array}{l}\text { Requested } \\
\text { Detection Limit }\end{array}$ \\
\hline \multicolumn{7}{|l|}{ Organic Compounds } \\
\hline & & & & & & \\
\hline Total Organic Carbon & $<2.00 \mathrm{E}+01$ & $<2.00 \mathrm{E}+01$ & $<2.00 \mathrm{E}+01$ & - & $5.00 \mathrm{E}+03$ & $1.00 \mathrm{E}-01$ \\
\hline Butanol \& Isobutanol & $<1.0 \mathrm{E}-01$ & $<1.0 \mathrm{E}-01$ & $<1.0 \mathrm{E}-01$ & - & $2.25 \mathrm{E}+03$ & $1.00 \mathrm{E}-01$ \\
\hline Isopropanol & $<5.0 \mathrm{E}-03$ & $<5.0 \mathrm{E}-03$ & $<5.0 \mathrm{E}-03$ & - & $2.25 \mathrm{E}+03$ & $1.00 \mathrm{E}-01$ \\
\hline Methanol & NA & NA & NA & - & $2.25 \mathrm{E}+02$ & NA \\
\hline Phenol & $<2.0 \mathrm{E}-02$ & $<2.0 \mathrm{E}-02$ & $<2.0 \mathrm{E}-02$ & - & $7.50 \mathrm{E}+02$ & $7.50 \mathrm{E}-01$ \\
\hline Tetraphenylborate (TPB) & $<2.30 \mathrm{E}-01$ & $<2.30 \mathrm{E}-01$ & $<2.30 \mathrm{E}-01$ & - & $3.00 \mathrm{E}+01$ & IDL \\
\hline Toluene & $<5.0 \mathrm{E}-03$ & $<5.0 \mathrm{E}-03$ & $<5.0 \mathrm{E}-03$ & - & $3.75 \mathrm{E}+02$ & $1.00 \mathrm{E}-02$ \\
\hline Tributylphsophate (TBP) & $<2.0 \mathrm{E}-02$ & $<2.0 \mathrm{E}-02$ & $<2.0 \mathrm{E}-02$ & - & $3.00 \mathrm{E}+02$ & $1.00 \mathrm{E}-02$ \\
\hline EDTA & $<1.5 \mathrm{E}+01$ & $<1.5 \mathrm{E}+01$ & $<1.5 \mathrm{E}+01$ & - & $3.75 \mathrm{E}+02$ & IDL \\
\hline Benzene & $<5.0 \mathrm{E}-03$ & $<5.0 \mathrm{E}-03$ & $<5.0 \mathrm{E}-03$ & - & - & - \\
\hline
\end{tabular}

$\mathrm{NA}=$ Not available

$\mathrm{IDL}=$ Instrument detection limit 
WSRC-TR-2005-00192, Rev. 1

May 5, 2005

Page 10 of 11

Table 2. Tank 23H WAC Sample Results for Radioactive Contaminants (Units of $\mathrm{pCi} / \mathrm{mL}$ )

\begin{tabular}{|c|c|c|c|c|c|c|}
\hline Radionuclide & Sample 1 & Sample 2 & Average & $\begin{array}{l}\text { Stand } \\
\text { deviation }\end{array}$ & WAC Limit & $\begin{array}{l}\text { Requested } \\
\text { Detection } \\
\text { Limit }\end{array}$ \\
\hline Tritium $\left({ }^{3} \mathrm{H}\right)$ & $1.00 \mathrm{E}+03$ & $1.05 \mathrm{E}+03$ & $1.03 \mathrm{E}+03$ & $3.54 \mathrm{E}+01$ & $5.63 \mathrm{E}+05$ & $1.00 \mathrm{E}+03$ \\
\hline Carbon-14 $\left({ }^{14} \mathrm{C}\right)$ & $4.26 \mathrm{E}+01$ & $3.96 \mathrm{E}+01$ & $4.11 \mathrm{E}+01$ & $2.07 \mathrm{E}+00$ & $1.13 \mathrm{E}+05$ & $1.00 \mathrm{E}+01$ \\
\hline Aluminum-26 $\left({ }^{26} \mathrm{Al}\right)$ & $<5.68 \mathrm{E}-02$ & $<6.26 \mathrm{E}-02$ & $<5.97 \mathrm{E}-02$ & - & $2.88 \mathrm{E}+03$ & $1.00 \mathrm{E}-01$ \\
\hline Nickel-59 $\left({ }^{59} \mathrm{Ni}\right)$ & $<1.22 \mathrm{E}+01$ & $<3.04 \mathrm{E}+01$ & $<2.13 \mathrm{E}+01$ & - & $1.13 \mathrm{E}+05$ & $1.00 \mathrm{E}-01$ \\
\hline Nickel-63 $\left({ }^{63} \mathrm{Ni}\right)$ & $<2.55 \mathrm{E}+01$ & $<4.95 \mathrm{E}+01$ & $<3.75 \mathrm{E}+01$ & - & $1.13 \mathrm{E}+05$ & $1.00 \mathrm{E}-01$ \\
\hline Cobalt-60 $\left({ }^{60} \mathrm{Co}\right)$ & $8.15 \mathrm{E}-01$ & $9.05 \mathrm{E}-01$ & $8.60 \mathrm{E}-01$ & $6.37 \mathrm{E}-02$ & $1.13 \mathrm{E}+06$ & IDL \\
\hline Selenium-79 $\left({ }^{79} \mathrm{Se}\right)$ & $<4.50 \mathrm{E}+00$ & $<1.15 \mathrm{E}+01$ & $<8.02 \mathrm{E}+00$ & - & $1.13 \mathrm{E}+05$ & $1.00 \mathrm{E}-01$ \\
\hline $\begin{array}{l}\text { Strontium/Yttrium-90 } \\
\left({ }^{90} \mathrm{Sr} / \mathrm{Y}\right)\end{array}$ & $2.66 \mathrm{E}+02$ & $3.74 \mathrm{E}+02$ & $3.20 \mathrm{E}+02$ & $7.64 \mathrm{E}+01$ & $2.87 \mathrm{E}+05$ & $1.00 \mathrm{E}+01$ \\
\hline Niobium-94 $\left({ }^{94} \mathrm{Nb}\right)$ & $<5.86 \mathrm{E}-01$ & $<6.49 \mathrm{E}-01$ & $<6.17 \mathrm{E}-01$ & - & $1.53 \mathrm{E}+04$ & $1.00 \mathrm{E}-01$ \\
\hline Technetium-99 (Tc-99) & $6.80 \mathrm{E}+01$ & $6.62 \mathrm{E}+01$ & $6.71 \mathrm{E}+01$ & $1.28 \mathrm{E}+00$ & $4.22 \mathrm{E}+05$ & $1.00 \mathrm{E}+01$ \\
\hline $\begin{array}{l}\text { Ruthenium/Rhodium-106 } \\
\left({ }^{106} \mathrm{Ru} / \mathrm{Rh}\right)\end{array}$ & $<1.45 \mathrm{E}+01$ & $<1.54 \mathrm{E}+01$ & $<1.49 \mathrm{E}+01$ & - & $1.13 \mathrm{E}+06$ & $1.00 \mathrm{E}+00$ \\
\hline Antimony-125 $\left({ }^{125} \mathrm{Sb}\right)$ & $<1.23 \mathrm{E}+01$ & $<1.32 \mathrm{E}+01$ & $<1.27 \mathrm{E}+01$ & - & $2.25 \mathrm{E}+06$ & $1.00 \mathrm{E}+00$ \\
\hline Tin-126 $\left({ }^{126} \mathrm{Sn}\right)$ & $<4.86 \mathrm{E}+00$ & $<5.14 \mathrm{E}+00$ & $<5.00 \mathrm{E}+00$ & - & $1.80 \mathrm{E}+04$ & $1.00 \mathrm{E}+00$ \\
\hline Iodine-129 $\left({ }^{129} \mathrm{I}\right)$ & $4.91 \mathrm{E}-01$ & $6.67 \mathrm{E}-01$ & $5.79 \mathrm{E}-01$ & $1.24 \mathrm{E}-01$ & $1.13 \mathrm{E}+03$ & $1.00 \mathrm{E}-02$ \\
\hline Cesium-134 $\left({ }^{134} \mathrm{Cs}\right)$ & $2.78 \mathrm{E}+00$ & $2.77 \mathrm{E}+00$ & $2.77 \mathrm{E}+00$ & $6.37 \mathrm{E}-03$ & $1.13 \mathrm{E}+06$ & IDL \\
\hline Cesium-135 $\left({ }^{135} \mathrm{Cs}\right)$ & $4.23 \mathrm{E}+00$ & $3.94 \mathrm{E}+00$ & $4.09 \mathrm{E}+00$ & $2.02 \mathrm{E}-01$ & $1.13 \mathrm{E}+06$ & IDL \\
\hline Cesium-137 $\left({ }^{137} \mathrm{Cs}\right)$ & $5.14 \mathrm{E}+04$ & $5.54 \mathrm{E}+04$ & $5.34 \mathrm{E}+04$ & $2.87 \mathrm{E}+03$ & $1.40 \mathrm{E}+06$ & $1.00 \mathrm{E}+03$ \\
\hline Cerium-144 $\left({ }^{144} \mathrm{Ce}\right)$ & $<1.18 \mathrm{E}+01$ & $<1.25 \mathrm{E}+01$ & $<1.21 \mathrm{E}+01$ & - & $1.13 \mathrm{E}+05$ & IDL \\
\hline Promethium-147 $\left({ }^{147} \mathrm{Pm}\right)$ & $<2.01 \mathrm{E}+00$ & $<2.85 \mathrm{E}+00$ & $<2.43 \mathrm{E}+00$ & - & $5.63 \mathrm{E}+06$ & IDL \\
\hline Samarium-151 $\left({ }^{151} \mathrm{Sm}\right)$ & $<2.90 \mathrm{E}+00$ & $<1.54 \mathrm{E}+00$ & $<2.22 \mathrm{E}+00$ & - & $2.25 \mathrm{E}+04$ & IDL \\
\hline Europium-154 $\left({ }^{154} \mathrm{Eu}\right)$ & $<9.41 \mathrm{E}-01$ & $<1.05 \mathrm{E}+00$ & $<9.93 \mathrm{E}-01$ & - & $2.25 \mathrm{E}+06$ & $1.00 \mathrm{E}-01$ \\
\hline Europium-155 $\left({ }^{155} \mathrm{Eu}\right)$ & $<2.67 \mathrm{E}+00$ & $<5.95 \mathrm{E}+00$ & $<4.31 \mathrm{E}+00$ & - & $1.13 \mathrm{E}+04$ & $1.00 \mathrm{E}-01$ \\
\hline Radium-226 $\left({ }^{226} \mathrm{Ra}\right)$ & $<2.55 \mathrm{E}+03$ & $<2.72 \mathrm{E}+03$ & $<2.64 \mathrm{E}+03$ & - & $8.73 \mathrm{E}+03$ & $1.00 \mathrm{E}+01$ \\
\hline Thorium-230 $\left({ }^{230} \mathrm{Th}\right)$ & $<6.96 \mathrm{E}+00$ & $<6.96 \mathrm{E}+00$ & $<6.96 \mathrm{E}+00$ & - & $1.62 \mathrm{E}+04$ & IDL \\
\hline Thorium-232 $\left({ }^{232} \mathrm{Th}\right)$ & $<3.62 \mathrm{E}-05$ & $<3.62 \mathrm{E}-05$ & $<3.62 \mathrm{E}-05$ & - & $2.88 \mathrm{E}+03$ & IDL \\
\hline Uranium-233 $\left({ }^{233} \mathrm{U}\right)$ & $<2.41 \mathrm{E}+00$ & $<2.41 \mathrm{E}+00$ & $<2.41 \mathrm{E}+00$ & - & $1.13 \mathrm{E}+04$ & IDL \\
\hline Uranium-234 $\left({ }^{234} \mathrm{U}\right)$ & $3.63 \mathrm{E}+00$ & $4.00 \mathrm{E}+00$ & $3.82 \mathrm{E}+00$ & $2.62 \mathrm{E}-01$ & $1.13 \mathrm{E}+04$ & $1.00 \mathrm{E}+00$ \\
\hline Uranium-235 $\left({ }^{235} \mathrm{U}\right)$ & $2.43 \mathrm{E}-02$ & $1.83 \mathrm{E}-02$ & $2.13 \mathrm{E}-02$ & $4.24 \mathrm{E}-03$ & $1.13 \mathrm{E}+02$ & $9.00 \mathrm{E}-03$ \\
\hline Uranium-236 $\left({ }^{236} \mathrm{U}\right)$ & $8.80 \mathrm{E}-02$ & $8.36 \mathrm{E}-02$ & $8.58 \mathrm{E}-02$ & $3.11 \mathrm{E}-03$ & $1.13 \mathrm{E}+04$ & $1.00 \mathrm{E}-02$ \\
\hline Uranium-238 $\left({ }^{238} \mathrm{U}\right)$ & $6.64 \mathrm{E}-01$ & $6.12 \mathrm{E}-01$ & $6.38 \mathrm{E}-01$ & $3.68 \mathrm{E}-02$ & $1.13 \mathrm{E}+04$ & $1.00 \mathrm{E}-01$ \\
\hline
\end{tabular}


WSRC-TR-2005-00192, Rev. 1

May 5, 2005

Page 11 of 11

Table 2. Tank 23H WAC Sample Results for Radioactive Contaminants (Units of $\mathbf{p C i} / \mathbf{m L}$ ) continued.

\begin{tabular}{|c|c|c|c|c|c|c|}
\hline Radionuclide & Sample 1 & Sample 2 & Average & $\begin{array}{l}\text { Stand } \\
\text { deviation }\end{array}$ & WAC Limit & $\begin{array}{l}\text { Requested } \\
\text { Detection } \\
\text { Limit }\end{array}$ \\
\hline Neptunium-237 $\left({ }^{237} \mathrm{~Np}\right)$ & $<1.77 \mathrm{E}-01$ & $<1.77 \mathrm{E}-01$ & $<1.77 \mathrm{E}-01$ & - & $2.25 \mathrm{E}+04$ & $1.00 \mathrm{E}-01$ \\
\hline Plutonium-238 $\left({ }^{238} \mathrm{Pu}\right)$ & $1.95 \mathrm{E}+02$ & $1.92 \mathrm{E}+02$ & $1.93 \mathrm{E}+02$ & $2.33 \mathrm{E}+00$ & $2.25 \mathrm{E}+04$ & $1.00 \mathrm{E}+00$ \\
\hline Plutonium-239 $\left({ }^{239} \mathrm{Pu}\right)$ & 8.42E-01 & 4.79E-01 & 6.62E-01 & $2.58 \mathrm{E}-01$ & $2.25 \mathrm{E}+04$ & $1.00 \mathrm{E}-01$ \\
\hline Plutonium-240 $\left({ }^{240} \mathrm{Pu}\right)$ & $2.11 \mathrm{E}-01$ & $1.20 \mathrm{E}-01$ & 1.65E-01 & 6.46E-02 & $2.25 \mathrm{E}+04$ & IDL \\
\hline Plutonium-241 $\left({ }^{241} \mathrm{Pu}\right)$ & $<2.17 \mathrm{E}+01$ & $<1.68 \mathrm{E}+01$ & $<1.93 \mathrm{E}+01$ & - & $8.38 \mathrm{E}+05$ & $1.00 \mathrm{E}+00$ \\
\hline Plutonium-242 $\left({ }^{242} \mathrm{Pu}\right)$ & $<9.83 \mathrm{E}-01$ & $<9.83 \mathrm{E}-01$ & $<9.83 \mathrm{E}-01$ & - & $2.25 \mathrm{E}+04$ & IDL \\
\hline Americium-241 $\left({ }^{241} \mathrm{Am}\right)$ & $2.55 \mathrm{E}+00$ & $2.50 \mathrm{E}+00$ & $2.53 \mathrm{E}+00$ & $3.82 \mathrm{E}-02$ & $2.25 \mathrm{E}+04$ & $1.00 \mathrm{E}+00$ \\
\hline Americium-243 $\left({ }^{243} \mathrm{Am}\right)$ & $<8.24 \mathrm{E}-02$ & $<6.08 \mathrm{E}-02$ & $<7.16 \mathrm{E}-02$ & - & $2.25 \mathrm{E}+04$ & IDL \\
\hline Curium-242 $\left({ }^{242} \mathrm{Cm}\right)$ & $<1.81 \mathrm{E}-03$ & $<1.59 \mathrm{E}-03$ & $<1.70 \mathrm{E}-03$ & - & $1.13 \mathrm{E}+04$ & IDL \\
\hline Curium-243 $\left({ }^{243} \mathrm{Cm}\right)$ & $<2.02 \mathrm{E}-01$ & $<1.51 \mathrm{E}-01$ & $<1.77 \mathrm{E}-01$ & - & - & - \\
\hline Curium-244 $\left({ }^{244} \mathrm{Cm}\right)$ & $1.03 \mathrm{E}-01$ & $4.55 \mathrm{E}-02$ & $7.41 \mathrm{E}-02$ & $4.05 \mathrm{E}-02$ & $2.25 \mathrm{E}+04$ & IDL \\
\hline Total Beta/Gamma & $7.66 \mathrm{E}+04$ & $7.25 \mathrm{E}+04$ & $7.45 \mathrm{E}+04$ & $2.87 \mathrm{E}+03$ & $2.37 \mathrm{E}+07$ & $1.00 \mathrm{E}+03$ \\
\hline Total Alpha & $7.03 \mathrm{E}+02$ & $6.22 \mathrm{E}+02$ & $6.62 \mathrm{E}+02$ & $5.73 \mathrm{E}+01$ & $2.50 \mathrm{E}+04$ & $1.00 \mathrm{E}+00$ \\
\hline Europium-152 $\left({ }^{152} \mathrm{Eu}\right)^{* *}$ & - & - & - & - & Total $\beta / \gamma$ bound & $1.00 \mathrm{E}+00$ \\
\hline Radium-228 $\left({ }^{228} \mathrm{Ra}\right) * *$ & - & - & - & - & Total $\beta / \gamma$ bound & $1.00 \mathrm{E}+00$ \\
\hline Actinium-227 $\left({ }^{227} \mathrm{Ac}\right){ }^{* *}$ & - & - & - & - & Total $\beta / \gamma$ bound & $1.00 \mathrm{E}+01$ \\
\hline Berkelium-249 $\left({ }^{249} \mathrm{Bk}\right) * *$ & - & & & & Total $\beta / \gamma$ bound & $4.29 \mathrm{E}+02$ \\
\hline Thorium-229 $\left({ }^{229} \mathrm{Th}\right) * *$ & - & - & - & - & Total $\alpha$ bounded & $1.00 \mathrm{E}+00$ \\
\hline $\begin{array}{l}\text { Protactinium-231 }\left({ }^{231} \mathrm{~Pa}\right) \\
* *\end{array}$ & - & - & - & - & Total $\alpha$ bounded & $1.00 \mathrm{E}+00$ \\
\hline Uranium-232 $\left({ }^{232} \mathrm{U}\right) * *$ & - & - & - & & Total $\alpha$ bounded & $1.00 \mathrm{E}+00$ \\
\hline Plutonium-244 $\left({ }^{244} \mathrm{Pu}\right)$ & $<1.0 \mathrm{E}-02$ & $<1.0 \mathrm{E}-02$ & $<1.0 \mathrm{E}-02$ & - & Total $\alpha$ bounded & $1.00 \mathrm{E}+00$ \\
\hline Curium-245 $\left({ }^{245} \mathrm{Cm}\right)$ & $<1.66 \mathrm{E}-01$ & $<1.25 \mathrm{E}-01$ & $<1.45 \mathrm{E}-01$ & - & Total $\alpha$ bounded & $1.00 \mathrm{E}+00$ \\
\hline Curium-247 $\left({ }^{247} \mathrm{Cm}\right)$ & $<1.69 \mathrm{E}-01$ & $<1.26 \mathrm{E}-01$ & $<1.48 \mathrm{E}-01$ & - & Total $\alpha$ bounded & $1.00 \mathrm{E}+00$ \\
\hline Curium-248 $\left({ }^{248} \mathrm{Cm}\right)$ & $<1.40 \mathrm{E}+00$ & $<1.40 \mathrm{E}+00$ & $<1.40 \mathrm{E}+00$ & - & Total $\alpha$ bounded & $1.00 \mathrm{E}+00$ \\
\hline Californium-249 $\left({ }^{249} \mathrm{Cf}\right)$ & $<1.78 \mathrm{E}-01$ & $<1.21 \mathrm{E}-01$ & $<1.50 \mathrm{E}-01$ & - & Total $\alpha$ bounded & $1.00 \mathrm{E}+00$ \\
\hline Californium-251 $\left({ }^{251} \mathrm{Cf}\right)$ & $<2.06 \mathrm{E}-01$ & $<1.45 \mathrm{E}-01$ & $<1.76 \mathrm{E}-01$ & - & Total $\alpha$ bounded & $1.00 \mathrm{E}+00$ \\
\hline Californium-252 $\left({ }^{252} \mathrm{Cf}\right)$ & $1.81 \mathrm{E}-03$ & $<1.59 \mathrm{E}-03$ & $<1.70 \mathrm{E}-03$ & - & Total $\alpha$ bounded & $1.00 \mathrm{E}+00$ \\
\hline
\end{tabular}

** Not analyzed for at the request of the customer although originally slated to be analyzed. 


\section{Distribution}

\begin{tabular}{|c|c|c|}
\hline B. H. & Culbertson & $704-27 \mathrm{~S}$ \\
\hline J. W. & Barber & 704-2H, Rm. 197 \\
\hline T. C. C & tandler, & $704-Z$ \\
\hline M. J. & Barnes & 773-A, Rm. B-132 \\
\hline W. M. & Barnes & 704-56H, Rm. 164 \\
\hline S. M. & Blanco & 766-H. Rm. 2434 \\
\hline L. R. & Bragg & 766-H, Rm. 2434 \\
\hline T. E. & Britt & 742-4G, Rm. 3 \\
\hline H. L. & Bui & 742-4G, Rm. 3 \\
\hline S. G. & Campbell & 703-H, Rm. 107 \\
\hline L. & Carey & 766-H, Rm. 2005A \\
\hline J. T. & Carter & 703-H, Rm 122 \\
\hline W. D. & Clark & 766-H, Rm. 2412 \\
\hline S. L. & Clifford & 766-H, Rm. 2443 \\
\hline J. J. & Connelly & 773-41A, Rm. 231 \\
\hline D. T. & Conrad & 766-H, Rm. 2007 \\
\hline J. W. & Ray & $704-S$ \\
\hline A. D. & Cozzi & 773-43A, Rm. 218 \\
\hline C. L. & Crawford & 773-41A, Rm. 180 \\
\hline D. A. & Crowley & 773-A, Rm. A-262 \\
\hline N. R. & Davis & 766-H, Rm. 1006 \\
\hline W. B. & Dean & 766-H, Rm. 2243 \\
\hline V. G. & Dickert & 703-H, Rm. 4 \\
\hline C. L. & Donahue & 241-162H, Rm. 6 \\
\hline M. D. & Drumm & 766-H, Rm. 2050 \\
\hline M. C. & Duff & 773-43A, Rm. 217 \\
\hline C. R. & Dyer & 766-H, Rm. 2426 \\
\hline R. E. & Eibling & 999-W, Rm. 335 \\
\hline G. N. & Eide & 241-121H, Rm. 6 \\
\hline H. H. & Elder & 703-H, Rm. 95 \\
\hline S. D. & Fink & 773-A, Rm. B-112 \\
\hline F. F. & Fondeur & 773-A, Rm. B-124 \\
\hline R. C. & Fowler & 703-H, Rm. 98 \\
\hline M.W. & Geeting & 766-H, Rm. 2035 \\
\hline B. A. & Gifford & 766-H, Rm. 1066D \\
\hline A. P. & Giordano & 703-H, Rm 79 \\
\hline J. C. & Griffin & 773-A, rm. A-231 \\
\hline H. D. & Harmon & 766-H, Rm. 2014 \\
\hline K. D. & Harp & 755-H, Rm. 1066B \\
\hline E. W. & Harrison & 766-H, Rm. 2034 \\
\hline K. A. & Hauer & 703-H, Rm. 11 \\
\hline D. L. & Hayes & 235-H, Rm. 134 \\
\hline D. T. & Herman & 735-11A, Rm. 104 \\
\hline P. J & Hill & 766-H, Rm. $1066 \mathrm{C}$ \\
\hline R. N. & Hinds & 766-H, Rm. 2430 \\
\hline D. T. & Hobbs & 773-A, Rm. B-117 \\
\hline E. W. & Holtzscheiter & 773-A, Rm. A-230 \\
\hline C. M. & Jantzen & 773-A, Rm. B-104 \\
\hline W. D. & Kerley & 766-H, Rm. 2010 \\
\hline E. T. & Ketusky & 703-H, Rm. 83 \\
\hline D. P. & Lambert & 773-A, Rm. B-132 \\
\hline C. A. & Lanigan & 766-H, Rm. 2440B \\
\hline C. A. & Langton & 773-43A, Rm. 219 \\
\hline T. T. & $\mathrm{Le}$ & 766-H, Rm. 2237 \\
\hline
\end{tabular}

(E)

(E)

(E)

(E)

(E)

(E)

(E)

(E)

(E)

(E)

(E)

(E)

(E)

(E)

(E)

(E)

(E)

(E)

(E)

(E)

(E)

(E)

(E)

(E)

(E)

(E)

(E)

(E)

(E, P)

(E)

(E)

(E)

(E)

(E)

(E)

(P)

(E)

(E)

(E)

(E)

(E)

(E)

(E)

(E)

(E)

(E)

(E)

(E)

(E)

(E)

(E)

(E)
R. K.

M. S.

D. B.

N. P.

J. C.

D. J.

K. B.

C. J.

G. A.

J. E.

D.

J. W.

L. T.

C. A.

L. M.

M. A.

M. R.

J. E.

L. D.

T. L.

L. M.

T. B.

J. A.

M. R.

S. H.

T. R.

M.A.

S. J.

B. C.

R. A.

P. J.

E.

S. C.

T. J.

R. H.

M. E.

W. E.

S. J.

S. G.

P. C.

G. A.

S. A.

H. Q.

J. E.

W. B.

D. D.

A. $\mathrm{O}$.

D. C.

G. G.

W. R.

G. C.

LWP File

STI

(E) Electronic,
Leugemors 766-H

704-S

703-H, Rm. 3

704-26F,Rm.11

773-42A,Rm. 173

742-4G, Rm. 5

773-42A,Rm.14

735-11A, Rm. 121

724-9E, Rm. 1

704-S

766-H, Rm. 2231

766-H, Rm. 2411

766-H, Rm. 2441

773-42A, Rm. 182

773-43A, Rm. 222

704-27S, Rm. 6

766-H, Rm. 2002

704-S, Rm. 18

703-H, Rm. 5

766-H, Rm. 2009

773-A, Rm. A-263

773-42A, Rm. 128

703-H, Rm. 99

773-42A, Rm. 123

703-H, Rm. 84

704-S, Rm. 65

Reynolds

766-H, Rm 2054

703-H, Rm. 126

Robertson

766-H, Rm. 2008

766-H, Rm. 2011

704-61S, Rm. 6

766-H, Rm. 2004

766-H, Rm. 2037

766-H, Rm. 2015

766-H, Rm. 2003

773-A, Rm. B-117

773-A, Rm. A-261

766-H, Rm. 2022

766-H, Rm. 2052

766-H, Rm. 2436

703-H, Rm. 96

766-H, Rm. 2016

766-H, Rm. 2232

704-S, Rm. 13 (E)

704-S, Rm. 16

773-A, Rm. B-124

766-H, Rm. 2423

704-S

773-A, Rm. B-129

773-42A, Rm. 171

766-H, Rm. 2024

773-42A

703-43A

(P) Paper Mail
(E)

(E)

(E)

(E)

(E)

(E)

(E)

(E)

(E)

(E)

(E)

(E)

(E)

(E)

(E)

(E)

(E)

(E)

(E)

(E)

(P)

(E)

(E)

(E)

(E)

(E)

(E)

(P)

(E)

(E)

(E)

(E)

(E)

(E)

(E)

(E)

(E)

(E)

(E)

(E)

(E)

(E)

(E)

(E)

(E)

(E)

(E, P)

(E) (P 3 copies)

* Our standard distribution format is electronic unless otherwise requested. 

